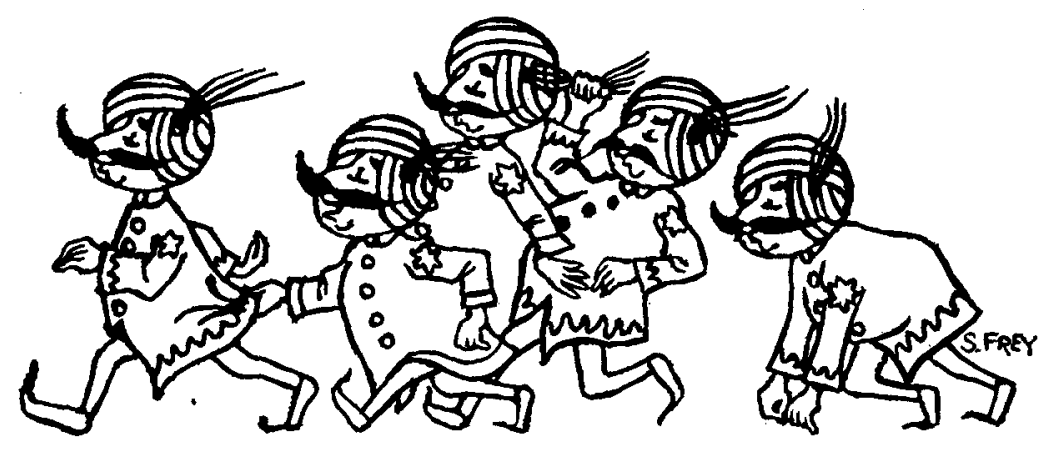

\title{
THE ONLY 747s FLYING EAST
}

AIR-INDIA Boeing 747s fly to New York from Paris, Frankfurt, Rome and London with very convenient connections from Geneva. Like other airlines. But unlike others, AIR-INDIA are the first to operate BOEING 747 FLIGHTS to the EAST. AIR-INDIA give passengers their first ever chance to fly eastwards on a Boeing 747 aircraft.

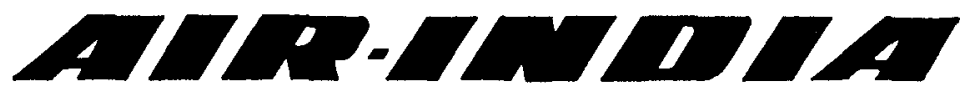

Geneva, 7, Chantepoulet, Phone (022) 320660 


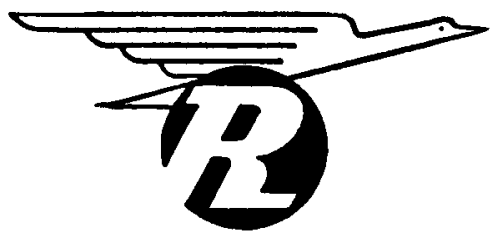

\section{H. Aitschard\&Cie.S.A}

\section{INTERNATIONAL TRANSPORT}

\section{TRAVEL AGENCY}

GENEVA, 49, route des Jeunes

Telephone 437600 - Teleprinter 22167

\section{Exchange - Tickets - Sea passages}

Insurance - Customs Agency

Road haulage - Storage

Home delivery of air and rail tickets on request bj telephone

Branches :

LAUSANNE - ANNEMASSE (France) 
AFGHANISTAN - Afghan Red Crescent, Puli Artan, Kabul.

ALBANIA - Albanian Red Cross, 35, Rruga e Barrikadavet, Tirana

ALGERIA - Algerian Red Crescent Society, 15 bis, Boulevard Mohamed V, Algiers.

ARGENTINA - Argentine Red Cross, H. Yrigoyen 2068, Buenos Aires.

AUSTRALIA - Australian Red Cross, 122 Flinders Street, Melbourne 3000.

AUSTRIA - Austrian Red Cross, 3 Gusshausstrasse, Postfach 39, Vienna 4.

BAHRAIN - Bahrain Red Crescent Society, P.O. Box 882, Manama.

BANGLADESH - Bangladesh Red Cross Society, Amin Court Building, Motijheel Commercial Area, Dacca 2. .

BELGIUM - Belgian Red Cross, 98 Chaussée de Vleurgat, 1050 Brussels.

BOLIVIA - Bolivian Red Cross, Avenida Simón Bolívar, 1515, La Paz.

BOTSWANA - Botswana Red Cross Society, Independence Avenue, P.O. Box 485, Gaborone.

BRAZIL - Brazilian Red Cross, Praça Cruz Vermelha 10-12, Rio de Janeiro.

BULGARIA - Bulgarian Red Cross, 1, Boul. Biruzov, Sofia 27.

BURMA (Socialist Republic of the Union of) Burma Red Cross, 42 Strand Road, Red Cross Building, Rangoon.

BURUNDI - Red Cross Society of Burundi, rue du Marché 3, P.O. Box 324, Bujumbura.

CAMBODIA - The new address of the Red Cross Society is not yet known.

CAMEROON - Cameroon Red Cross Society, rue Henry-Dunant, P.O.B. 631, Yaounde.

CANADA - Canadian Red Cross, 95 Wellesley Street East, Toronto, Ontario, M4Y 1 H6.

CENTRAL AFRICAN REPUBLIC - Central African Red Cross, B.P. 1428, Bangui.

CHILE - Chilean Red Cross, Avenida Santa María 0150, Correo 21, Casilla 246V., Santiago de Chile.

CHINA - Red Cross Society of China, 22 Kanmien Hutung, Peking, $E$.

COLOMBIA - Colombian Red Cross, Carrera 7a, 34-65, Apartado nacional 1110, Bogotá D.E.

COSTA RICA - Costa Rican Red Cross, Calle 14, Avenida 8, Apartado 1025, San José.

CUBA - Cuban Red Cross, Calle 23201 esq. N. Vedado, Havana.

CZECHOSLOVAKIA -- Czechoslovak Red Cross, Thunovska 18, 11804 Prague I.

DAHOMEY - Dahomean Red Cross, P.O. Box 1, Porto Novo.

DENMARK - Danish Red Cross, Ny Vestergade 17, DK-1471 Copenhagen $K$.

DOMINICAN REPUBLIC - Dominican Red Cross, Apartado Postal 1293, Santo Domingo.

ECUADOR - Ecuadorian Red Cross, Calle de la Cruz Roja y Avenida Colombia, 118, Quito.

EGYPT (Arab Republic of) - Egyptian Red Crescent Society, 34 rue Ramses, Cairo.

EL SALVADOR - El Salvador Red Cross, 3a Avenida Norte y 3a Calle Poniente, San Salvador, C.A.

ETHIOPIA - Ethiopian Red Cross, Ras Desta Damtew Avenue, Addis Ababa.
FIJI - Fiji Red Cross Society, 193 Rodwell Road, P.O. Box 569, Suva.

FINLAND - Finnish Red Cross, Tehtaankatu 1 A, Box 168, 00141 Helsinki 14.

FRANCE - French Red Cross, 17 rue Quentin Bauchart, F-75384 Paris, CEDEX 08.

GAMBIA - The Gambia Red Cross Society, P.O. Box 472, Banjul.

GERMAN DEMOCRATIC REPUBLIC - German Red Cross in the German Democratic Republic, Kaitzerstrasse 2, DDR 801 Dresden 1.

GERMANY, FEDERAL REPUBLIC OF - German Red Cross in the Federal Republic of Germany, Friedrich-Ebert-Allee 71, 5300, Bonn 1, Postfach (D.B.R.).

GHANA - Ghana Red Cross, National Headquarters, Ministries Annex A3, P.O. Box 835, Accra.

GREECE - Hellenic Red Cross, rue Lycavittou 1, Athens 135.

GUATEMALA - Guatemalan Red Cross, 3a Calle 8-40, Zona 1, Ciudad de Guatemala.

GUYANA - Guyana Red Cross, P.O. Box 351, Eve Leary, Georgetown.

HAITI - Haiti Red Cross, Place des Nations Unies, B.P. 1337, Port-au-Prince.

HONDURAS - Honduran Red Cross, 1a Avenida entre 3a y 4a Calles, № 313, Comayagüela, D.C.

HUNGARY - Hungarian Red Cross, V. Arany János utca 31, Budapest V. Mail Add.: 1367 Budapest 5, Pf. 249.

ICELAND - Icelandic Red Cross, Nóatúni 21, Reykjavik.

INDIA - Indian Red Cross, 1 Red Cross Road, New Delhi 110001 .

INDONESIA - Indonesian Red Cross, Jalan Abdul Muis 66, P.O. Box 2009, Djakarta.

IRAN - Iranian Red Lion and Sun Society, Av. Villa, Carrefour Takhté Djamchid, Teheran.

IRAQ - Iraqi Red Crescent, Al-Mansour, Baghdad.

IRELAND - Irish Red Cross, 16 Merrion Square, Dublin 2.

ITALY - Italian Red Cross, 12 via Toscana, Rome.

IVORY COAST - Ivory Coast Red Cross Society, B.P. 1244, Abidjan.

JAMAICA - Jamaica Red Cross Society, 76 Arnold Road, Kingston 5.

JAPAN — Japanese Red Cross, 29-12 Shiba 5-chome, Minato-Ku, Tokyo 108.

JORDAN - Jordan National Red Crescent Society, P.O. Box 10001 , Amman.

KENYA - Kenya Red Cross Society, St. John's Gate, P.O. Box 40712, Nairobi.

KOREA, DEMOCRATIC PEOPLE'S REPUBLIC $O F$ - Red Cross Society of the Democratic People's Republic of Korea, Pyongyang.

KOREA, REPUBLIC OF - The Republic of Korea National Red Cross, 32-3Ka Nam San-Dong, Seoul.

KUWAIT - Kuwait Red Crescent Society, P.O. Box 1350, Kuwait.

LAOS - Lao Red Cross, P.B. 650, Vientiane.

LEBANON - Lebanese Red Cross, rue Général Spears, Beirut.

LESOTHO - Lesotho Red Cross Society, P.O. Box 366, Maseru. 
LIBERIA - Liberian National Red Cross, National Headquarters, 107 Lynch Street, P.O. Box 226, Monrovia.

LIBYAN ARAB REPUBLIC - Libyan Arab Red Crescent, P.O. Box 541, Benghazi.

LIECHTENSTEIN - Liechtenstein Red Cross, Vaduz.

LUXEMBOURG - Luxembourg Red Cross, Parc de la Ville, C.P. 1806, Luxembourg.

MALAGASY REPUBLIC - Red Cross Society of the Malagasy Republic, rue Clémenceau, P.O. Box 1168, Tananarive.

MALAWI - Malawi Red Cross, Hall Road, Blantyre (P.O. Box 30080, Chichiri, Blantyre 3).

MALAYSIA - Malaysian Red Crescent Society, 519 Jalan Belfield, Kuala Lumpur 08-03.

MALI - Mali Red Cross, B.P. 280, route de Koulikora, Bamako.

MAURITANIA - Mauritanian Red Crescent Society, B.P. 344, Avenue Gamal Abdel Nasser, Nouakchott.

MEXICO - Mexican Red Cross, Avenida Ejército Nacional no 1032, México 10 D.F.

MONACO - Red Cross of Monaco, 27 boul. de Suisse, Monte Carlo.

MONGOLIA - Red Cross Society of the Mongolian People's Republic, Central Post Office, Post Box 537, Ulan Bator.

MOROCCO - Moroccan Red Crescent, B.P. 189, Rabat.

NEPAL - Nepal Red Cross Society, Tahachal, P.B. 217, Kathmandu.

NETHERLANDS - Netherlands Red Cross, 27 Prinsessegracht, The Hague.

NEW ZEALAND - New Zealand Red Cross, Red Cross House, 14 Hill Street, Wellington 1. (P.O. Box 12-140, Wellington North.)

NICARAGUA - Nicaraguan Red Cross, Managua, D.N.

NIGER - Red Cross Society of Niger, B.P. 386, Niamey.

NIGERIA - Nigerian Red Cross Society, Eko Aketa Close, off St. Gregory Rd., P.O. Box 764, Lagos.

NORWAY - Norwegian Red Cross, Parkveien 33b, Oslo. Mail Add.: Postboks $7034 \mathrm{H}$-Oslo 3.

PAKISTAN - Pakistan Red Crescent Society, Dr Daudpota Road, Karachi 4.

PANAMA - Panamanian Red Cross, Apartado Postal 668, Zona 1, Panamá.

PARAGUAY - Paraguayan Red Cross, Brasil 216, Asuncion.

PERU - Peruvian Red Cross, Jirón Chancay 881, Lima.

PHILIPPINES - Philippine National Red Cross, 860 United Nations Avenue, P.O.B. 280, Manila $D-406$.

POLAND - Polish Red Cross, Mokotowska 14, Warsaw.

PORTUGAL - Portuguese Red Cross, Jardim 9 de Abril, 1 a 5, Lisbon 3.

ROMANIA - Red Cross of the Socialist Republic of Romania, Strada Biserica Amzei 29, Bucarest.

SAN MARINO - San Marino Red Cross, Palais gouvernemental, San Marino.

SAUDI ARABIA - Saudi Arabian Red Crescent, Riyadh.
SENEGAL - Senegalese Red Cross Society, Bd Franklin-Roosevelt, P.O.B. 299, Dakar.

SIERRA LEONE - Sierra Leone Red Cross Society, 6A Liverpool Street, P.O.B. 427, Freetown.

SINGAPORE - Singapore Red Cross Society, 15 Penang Lane, Singapore 9.

SOMALI REPUBLIC - Somali Red Crescent Society, P.O. Box 937, Mogadishu.

SOUTH AFRICA - South African Red Cross, Cor. Kruis \& Market Streets, P.O.B. 8726, Johannesburg 2000.

SPAIN - Spanish Red Cross, Eduardo Dato 16, Madrid 10.

SRI LANKA - Sri Lanka Red Cross Society, 106 Dharmapala Mawatha, Colombo 7.

SUDAN - Sudanese Red Crescent, P.O. Box 235, Khartoum.

SWEDEN - Swedish Red Cross, Fack, S-104 40 Stockholm 14.

SWITZERLAND - Swiss Red Cross, Taubenstrasse 8, B.P. 2699, 3001 Berne.

SYRIAN ARAB REPUBLIC - Syrian Red Crescent, Bd Mahdi Ben Barake, Damascus.

TANZANIA - Tanzania Red Cross Society, Upanga Road, P.O.B. 1133, Dar es Salaam.

THAILAND - Thai Red Cross Society, Paribatra Building, Chulalongkorn Memorial Hospital, Bangkok.

TOGO - Togolese Red Cross Society, 51 rue Boko Soga, P.O. Box 655, Lomé.

TRINIDAD AND TOBAGO … Trinidad and Tobago Red Cross Society, Wrightson Road West, P.O. Box 357, Port of Spain, Trinidad, West Indies.

TUNISIA - Tunisian Red Crescent, 19 rue d'Angleterre, Tunis.

TURKEY - Turkish Red Crescent, Yenisehir, Ankara.

UGANDA - Uganda Red Cross, Nabunya Road, P.O. Box 494, Kampala.

UNITED KINGDOM - British Red Cross, 9 Grosvenor Crescent, London, SWIX $7 E J$.

UPPER VOLTA - Upper Volta Red Cross, P.O.B. 340, Ouagadougou.

URUGUAY - Uruguayan Red Cross, Avenida 8 de Octubre 2990, Montevideo.

U.S.A. - American National Red Cross, 17th and D Streets, N.W., Washington, D.C. 20006.

U.S.S.R. - Alliance of Red Cross and Red Crescent Societies, Tcheremushki, I. Tcheremushkinskii proezd 5, Moscow B-36.

VENEZUELA - Venezuelan Red Cross, Avenida Andrés Bello No. 4, Apart. 3185, Caracas.

VIET NAM, DEMOCRATIC REPUBLIC OF Red Cross of the Democratic Republic of Viet Nam, 68 rue Bà-Trièu, Hanoi.

SOUTH VIET NAM - Red Cross of the Republic of South Viet Nam, Hông-Thâp-Tu street, 201, Saigon.

YUGOSLAVIA - Red Cross of Yugoslavia, Simina ulica broj 19, Belgrade.

ZAIRE (Republic of) - Red Cross of the Republic of Zaire, 41 av. de la Justice, B.P. 1712, Kinshasa.

ZAMBIA - Zambia Red Cross, P.O. Box R.W.1, 2837 Brentwood Drive, Lusaka. 